\title{
Near-field observation of evanescent light wave coupling in subwavelength optical waveguides
}

\author{
R. Quidant ${ }^{1}$, J.-C. Weeber ${ }^{1}$, A. Dereux ${ }^{1}$, D. Peyrade ${ }^{2}$, Y. Chen $^{2}$ and C. \\ GIRARD $^{3}$ \\ 1 Laboratoire de Physique de l'Université de Bourgogne - Optique Submicronique, 9 av- \\ enue A. Savary, F-21078 Dijon, France \\ 2 Laboratoire de Microstructures et Microélectronique (CNRS) - 196 Avenue H. Ravera, \\ F-92225 Bagneux, France \\ 3 Centre d'Elaboration des Matériaux et d'Etudes Structurales (CNRS) - 29 rue J. Mar- \\ vig, F-31055 Toulouse, France
}

PACS. 42.79. Gn - Optical waveguides and couplers.

PACS. 42.82.Et - Waveguides, couplers, and arrays.

PACS. 07.79.Fc - Near-field scanning optical microscopes.

\begin{abstract}
We report the observation, in the range of visible frequencies, of the coupling of light into integrated waveguides of subwavelength cross-sections together with a subwavelength detection at the output of such guides. Coupling in is produced by controlling the focusing of a laser beam totally reflected at the surface of the sample. Several tens of micrometres long dielectric ridges have been efficiently excited with this technique. The phenomenon is observed in direct space by a Photon Scanning Tunneling Microscope which also allows to test the principle of detection in a subwavelength volume.
\end{abstract}

In modern optical communication technologies, transferring optical signals is often achieved by an optical waveguide connexion [1]. When integrated in planar geometry, such devices can guide optical beams with low losses over several hundreds of micrometres. But, if the cross section $\sigma$ of microfabricated waveguides is scaled below the diffraction limit $(\lambda \leq \sqrt{\sigma}$, where $\lambda$ is the incident wavelength), the following specific difficulties arise:

(i) An efficient coupling of light into such an optical waveguide requires a perfect alignment of the wavevector of the incoming wave with the longitudinal axis of the guide.

(ii) Near the diffraction limit, the drastic decrease of available electromagnetic eigenmodes of the waveguide makes the use of standard optical couplers difficult. Direct focusing, prisms and grating couplers are commonly used techniques. However, in the visible range, such couplers cannot be easily applied to structures with submicrometre transverse sections.

(iii) Last but not least, the detection at the exit of a waveguide with a sub- $\lambda$ cross section (shortened to "sub- $\lambda$ waveguide") is facing one consequence of the Heisenberg uncertainty principle. Indeed, if one intends to keep a practical sense to the miniaturization of optical connexions, the sizes of the detection volumes should be commensurable with the transverse sizes of the sub- $\lambda$ waveguides. In other words, a detection volume featuring supra- $\lambda$ sizes at 
the exit of a sub- $\lambda$ waveguide downgrades the practical value of such guides for miniaturization purposes. However, for an electromagnetic wave, Heisenberg demonstrated [2] that an uncertainty relation involves the Cartesian components of the electric $\left(E_{x}, E_{y}, E_{z}\right)$ and the magnetic $\left(H_{x}, H_{y}, H_{z}\right)$ fields vectors (SI units, $c$ is the speed of light in vacuum and $h$ is the Planck constant):

$$
\Delta E_{x} \Delta H_{y} \geq \frac{h c^{2}}{4 \pi(\delta l)^{4}},
$$

with cyclic permutation for the other Cartesian components. These uncertainty relations refer to a simultaneous knowledge (in the sense of quantum theory) of the electric and magnetic fields in the same volume $(\delta l)^{3}$. In the case of optical frequencies, it implies that sub $-\lambda$ detection can provide information on the electric field or on the magnetic field but not on both of them simultaneously. Since the energy of an electromagnetic wave sums the electric and magnetic contributions, performing the usual energy measurement in a subwavelength volume is directly concerned by the consequence of the above uncertainty relation. Therefore, flares at the output widen sub- $\lambda$ waveguides (up to ten times) in order to recover an energy measurement in a supra $-\lambda$ volume which remains unaffected by the Heisenberg uncertainty [3].

The present work explores new routes to overpass the difficulties arising from these restrictive items. Solutions are proposed to achieve the efficient optical addressing of integrated waveguides featuring: (a) transverse sizes ranging in the subwavelength domain (down to $\sigma$ $\simeq 150 \times 200 \mathrm{~nm}^{2}$ ); (b) modes confined laterally within a width of about the half of the incident wavelength; (c) demonstration of detection in a subwavelength volume at the exit of the guide. To handle the difficulty (i), the basic idea consists in the use of mostly evanescent optical fields instead of the common propagating laser beams. Such a state of the incident field can be produced by three-dimensional (3D) focused Gaussian beams which are incident in the transparent substrate in such a way that it is totally reflected when there is not any structure deposited on the surface of the said substrate. Recently, the pertinence of this coupling mode was checked on various configurations by extensive numerical simulations based on the Green dyadic technique [4]. To overcome the difficulty (ii), the reduction of the transverse section of a waveguide has to be balanced by increasing the contrast of dielectric functions between the constitutive material and the surrounding media, namely the air and the glass substrate in our case [5]. With a dielectric function of about 5.3, $\mathrm{TiO}_{2}$ allows to reach our purpose. To demonstrate the detection in a subwavelength volume which avoids the uncertainty on energy measurements (difficulty (iii)), one should rather rely on a measurement of the electric or the magnetic field intensity. For this purpose, we used a Photon Scanning Tunneling Microscope (PSTM) [7]. When operated at constant height, it has been shown that this device performs one or the other of these measurements $[8,9]$.

In addition to providing a subwavelength detection volume at the exit of the guide, this configuration of scanning near-field optical microscope can also characterize, in direct space and with subwavelength resolution, the optical properties of nanostructures integrated in coplanar geometry [10,11]. Specifically, the PSTM turned out to be well-suited to map the propagation of light at the submicrometre scale [12].

A sample was obtained by standard electron beam lithography and reactive ion etching techniques [6]. A planar BK7 glass substrate (refraction index: 1.515) was first coated with a $150 \mathrm{~nm}$ thick $\mathrm{TiO}_{2}$ layer. The resulting sample consists in a planar glass surface supporting several Subwavelength Optical Waveguides (SOW) that all display the same geometrical parameters (40 $\mu \mathrm{m}$ long, $200 \mathrm{~nm}$ wide and $150 \mathrm{~nm}$ high). The spacing $(\simeq 20 \mu \mathrm{m})$ between each SOW was chosen sufficiently large to prevent any coupling between them. 
This sample was then mounted on the glass prism (refraction index: 1.515) of a PSTM (cfr. figure (1)). In a PSTM, a pointed tip is piezo-electrically driven to scan close to the sample surface so as to act as a local probe of the optical field in the near-field zone. In this work, the sharply elongated tips were obtained by pulling an optical fiber which was subsequently coated with $7 \mathrm{~nm}$ of $\mathrm{Cr}$ (no resulting aperture). This coating shields the direct illumination of the fibre tip by scattered light, so that waves propagating in the $z$ direction (fig. 1) do not contribute significantly to the detected signal. The PSTM was scanning at constant height. In this mode of operation, the signal detected by a PSTM, equipped with such tip, is known to be related to the spatial distribution of the intensity of the optical electric field in the near-field zone $\left(I_{n f}\right)[8,9]$.

For our purpose, the specific feature of the PSTM setup is the incident field: instead of the usual extended plane wave, we used a focused Gaussian beam which is totally reflected at the substrate interface. This local illumination is produced by a He-Ne laser (wavelength in vacuum: $633 \mathrm{~nm}$ ) injected in a lensed single mode fibre (output power: $2 \mathrm{~mW}$ ). This fibre, not to be confused with the detecting fibre tip, shines the light through the substrate under the conditions of total internal reflection. The extremity of the fiber is oriented in such a way that the beam can be focused at the interface between the dielectric ridge and the glass substrate. We only considered the case of a transverse magnetic (TM) polarized incident beam (electric field in the plane of incidence) where, on the basis of numerical simulations, the coupling is expected to be more efficient. When illuminating the glass substrate with an angle of incidence of 50 degrees, the PSTM clearly identifies a central Gaussian peak in which the maximum of intensity is localized inside an area of $10 \times 5 \mu \mathrm{m}^{2}$. At higher detection planes, the signal detected by the tip decreases drastically. Clear identification of such a spot is thus only possible by using a PSTM. The diffraction rings are due to aberration effects on the prism interface. They are significantly pronounced at the "backfront" of the central peak. It will turn out that they do not downgrade the ability of the said central peak to address the extremity of any SOW deposited on the sample surface.

When shining a Gaussian beam in the total internal reflection setup used here, one may expect to exploit the Goos-Hänschen shift which results in the fact that the incident and reflected beams are not symmetrical with respect to the focal point [13]. Of course, in the air side of the air-glass interface, a wave vector parallel to the surface of the substrate is associated with this effect. The Gaussian beam is now focused at the right extremity of the SOW in such a way that the components of the incident wave vector parallel to the surface of the substrate be aligned along the longitudinal axis of the SOW (figure 2a). This condition is much easier to meet than the alignement of all three components of the incident wavevector, thereby overcoming the difficulty (i) listed above.

When recording PSTM images, in order to avoid any ambiguity, we did not use any feedback loop relying on any auxiliary force to control the motion of the tip: the tip scans in a plane above the sample surface while monitoring the light intensity level. Successive images were recorded when approaching the tip closer and closer to the surface of the sample. In the image presented in figure $(2 \mathrm{a})$ the constant height of the scan was fixed at about $\simeq 500 \pm 150$ $\mathrm{nm}$ above the substrate, i.e at about $350 \pm 150 \mathrm{~nm}$ above the top of the SOW. Since there is not any auxiliary force control of the tip, in order to avoid crashing the tip, the height of scan was not reduced anymore when, like in figures 2 (a-c), scanning is performed over such larges distances as $60 \mu \mathrm{m}$. Indeed, a loss of parallelism as slight as 0.15 degrees leads to a height difference of about $150 \mathrm{~nm}$ after scanning over $60 \mu \mathrm{m}$. Anyway, within this height range, in spite of the strong intensity delivered by the Gaussian beam (which defines the range of the intensity scale), we observe clearly a guiding process along the ridge and a light spot at the "exit" end of the SOW, on the left side of figure $2 \mathrm{a}$. We have reproduced, without any 
difficulty, similar effects on more than 30 different dielectric ridges.

To assess the role of the Goos-Hänschen shift in the efficiency of the coupling technique, we have studied the influence of the incident spot position with respect to the SOW "entry" end. Figures ( $2 \mathrm{~b}$ and $\mathrm{c}$ ) display two other illumination configurations realized successively on the same structure. First, figure $2 \mathrm{~b}$ shows the influence of the longitudinal spot position on the coupling efficiency. The extinction of the spot at the exit end of the SOW indicates that no coupling occurs anymore if the incident beam is located beyond $6 \mu \mathrm{m}$ to the right of the previous optimal configuration. In this case, all the incident wave is reflected back in the prism before reaching the structure. Similarly, the coupling does not occur when the incident spot position is moved beyond a distance of $8 \mu \mathrm{m}$ in the direction of positive values of $y$ (cf. figure 2c) or in the direction of negative values of $y$ (not shown for the sake of brevity).

In order to improve the visualization of the light localization phenomenon inside the SOW, one has to get rid of the above mentionned problem of parallelism between the tip trajectory and the surface of the substrate. This is possible by performing successively five overlapping PSTM images of size $5 \times 5 \mu \mathrm{m}^{2}$ recorded above the last quarter of the SOW by keeping the distance between the tip and the top of the SOW between 10 and $50 \mathrm{~nm}$. The corresponding result is gathered in figure (3). In order to visualize the lower intensity of the guiding process, the scale of the left image of figure (3) was truncated to match the intensity range of the other four images. Therefore, this left image of 3 does not detail the precise shape of the light spot that tails off the excited SOW. On the original scale, this spot appears as intense as 1.5 and is well localized with an FWHM area of about $0.4 \times 0.4 \mu \mathrm{m}^{2}$. As pointed out by the Heisenberg uncertainty mentionned above, a sharp measurement of the intensity profile of the electric field by the subwavelength volume of the probe tip prevents to measure a sharp energy profile at the exit end of the waveguide. Assessing the efficiency of the energy transfer on the basis of this spot is therefore not possible. Nevertheless, a detection process based on the measurement of the electric field intensity turns out to be feasible.

In spite of the subwavelength transverse sizes of the SOW, the mode remains well confined inside the structure. The full width at half maximum of the spatial distribution of the intensity of the mode along the transverse $(y)$ direction is found to be around $300 \mathrm{~nm}$ (cf. figure 4). Again, a clear identification of this mode is only possible by using a PSTM. This value is found to be in good agreement with the theoretical assessment of similar SOW's [4] which anticipated the experimental work reported here. To describe SOW's theoretically, reference [4] applied the Green's dyadic method $[14,15]$ to compute the electromagnetic near-field distribution in a plane parallel to the sample surface, when it is illuminated with an evanescent Gaussian beam [16]. We repeated the computations of reference [4] for the parameters of the section of the SOW studied here but, in order to keep a reasonable calculation time, the length was reduced to $10 \mu \mathrm{m}$. The modelling assumed a perfect rod of $\mathrm{TiO}_{2}$ (optical index 2.3) supported by a half space filled of glass (optical index 1.5).

In figure 4 , a cross-cut of the the numerical output (dashed line) is compared with a crosscut of the experimental signal (solid line) along the dashed line of figure (3). Such cross-cut provides an image of the width in direct space of the excited mode. In both experimental and theoretical patterns, a single maximum shows up, suggesting that the propagation along the SOW is supported by the lowest frequency mode that can be sustained by the SOW.

Except a less pronounced modulation of the field intensity along the SOW, we noticed a striking analogy between figure (3) and the numerical simulation displayed in the figure $3 \mathrm{~A}$ of reference [4]. In fact, a cross-cut along the longitudinal $(x)$ axis of the SOW in the calculated image would exhibit a standing wave pattern which does not show up so sharply in the experiment. This standing wave pattern arises due to the interferences of the incoming light with the light reflected back at the end of the SOW. The topography of the sample, as recorded 
by an Atomic Force Microscope (not shown for the sake of brevity), reveals a significant roughness $(\simeq 15 \mathrm{~nm})$ at the surface of the $\mathrm{TiO}_{2}$ wires. The resulting random scattering probably produces the observed more fuzzy standing wave pattern. A closer examination of the data following a longitudinal cross-cut along the SOW reveals a periodicity of $220 \mathrm{~nm}$ corresponding to an effective index of the guided mode of about 1.44 .

In order to show that SOW can open the way to optical circuitry with a resolution below $300 \mathrm{~nm}$, we designed a splitter (figure 5 (top)) which meets the practical requirement for high density optical connexions. The PSTM measurement (figure 5 (bottom))shows that the light is efficiently splitted within a short distance without significant losses at the junction. Also note the absence of any crosstalk even for very small spacing between the two branches of the junction.

In conclusion, the experiments described in this paper clearly demonstrate that dielectric ridges, featuring both transverse sizes in the subwavelength range, can guide light at visible frequencies. The guiding process relies on the lowest frequency mode sustained by the device. Such mode can be excited locally at the "entry" end of the SOW by focusing a totally reflected Gaussian beam and detected, in a subwavelength volume, at the "exit" end using a detection process which does not rely on an energy measurement, thereby avoiding the consequences of the Heisenberg uncertainty. This non standard coupling technique is efficient to excite narrow waveguides in coplanar geometry, opening the way to optical circuitry with a resolution below $300 \mathrm{~nm}$. In agreement with theoretical predictions, PSTM images have revealed, in the direct space, efficient transfers occurring over several tens of microns and confined laterally within a width of about the half of the incident wavelength. Finally, the well-localized light spots observed at the SOW "exit" could be used to address optically nanoscopic objects deposited on a surface.

This work was funded by the Regional Council of Burgundy and by the French Ministry for Education and Research through the program "Nanostructures et Fonctionnalités".

\section{REFERENCES}

[1] Burns W. K. and Milton A. F., Guided-wave optoelectronics, edited by Tamir T. (Springer, Berlin) 1988.

[2] Heisenberg W., The physical principles of the quantum theory (University of Chicago Press) 1930.

[3] Foresi J. S. et al., Nature, 390 (1997) 143.

[4] Weeber J. C. et al., Phys. Rev. E, 62 (2000) 738.

[5] Girard C., Dereux A., and Joachim C., Europhys. Lett., 44 (1998) 686.

[6] Lalanne P. et al., J. Opt. A: Pure App. Opt., 10 (1999) 215.

[7] Redilck R. C., Warmack R. J., and Ferrell T. L., Phys. Rev. B, 39 (1989) 767.

[8] Devaux E. et al., Phys. Rev. B, 62 (2000) 10504.

[9] Dereux A., Girard C. and Weeber J. C., J. Chem. Phys., 112 (2000) 7775.

[10] Balistreri M. L. M. et al., Phys. Rev. Lett., 85 (2000) 294.

[11] Chоo A. G. et al, Appl. Phys. Lett., 65 (1994) 947.

[12] Krenn J. R. et al., Phys. Rev. Lett., 82 (1999) 2590.

[13] Kosaki S. and Sakurai H., J. Opt. Soc. Am., 68 (1978) 508.

[14] Girard C. and Dereux A., Rep. Prog. Phys., 59 (1996) 657.

[15] Martin O. J. F., Girard C., and Dereux A., Phys. Rev. Lett., 74 (1995) 526.

[16] Baida F. I., Van Labeke D. and J. M. Vigoureux, Phys. Rev. B, 60 (1999) 7812. 


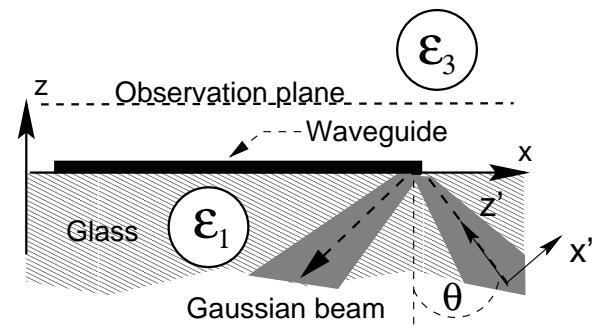

Fig. 1 - Geometry of the device. A SOW of $\mathrm{TiO}_{2}$ lying on the surface of a glass substrate (dielectric function $\varepsilon_{1}$ ) is illuminated by a Gaussian beam reflected at the interface between the substrate and the upper medium (air, dielectric function $\varepsilon_{3}$ ). The near-field optical detection is achieved with the tip of a PSTM scanning the observation plane.
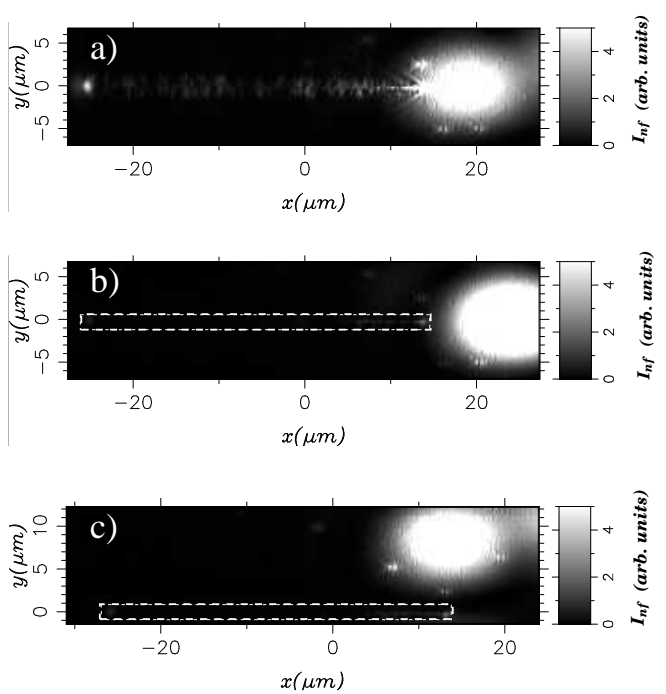

Fig. 2 - Near-field optical response of a SOW coupled with a totally reflected focused Gaussian beam (incident wavelength in vacuum $633 \mathrm{~nm}$, angle of reflection angle of $50^{\circ}$ ). The influence of the beam position is investigated: a) optimal configuration, b) $6 \mu \mathrm{m}$ longitudinal shift, c) $8 \mu \mathrm{m}$ transversal shift. In b) and c), the dotted-dashed lines show the position of the SOW.

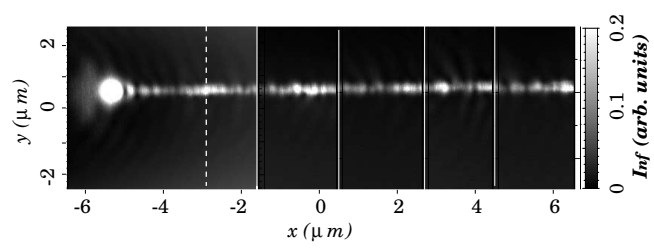

Fig. 3 - Assembling of five $5 \mu \mathrm{m} \times 5 \mu \mathrm{m}$ PSTM images recorded above the last quarter of the SOW. The solid vertical lines show the boundaries of the overlapping images. The dashed line locates the cross-cut displayed in figure 5. The left image, where a spot shows up, is recorded above the "exit" end of the SOW and its instensity scale as been truncated (see text). 


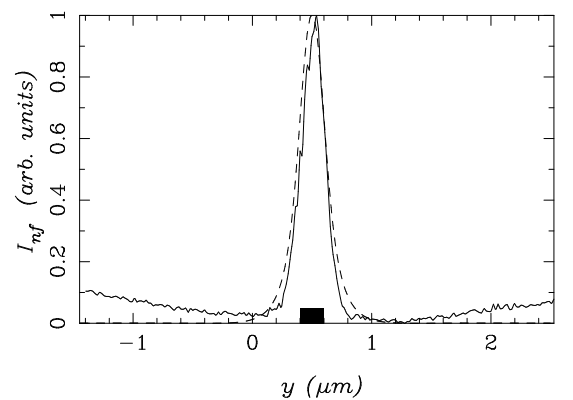

Fig. 4 - Theoretical (dashed line) and experimental (solid line) cross-cuts of the intensity of the optical electric field above the excited SOW. The calculation has been performed at $100 \mathrm{~nm}$ above the top of the SOW. The experimental cross-cut follows the dashed line of figure (3). As a guide to the eyes, the black area indicates the position of the SOW along the cross-cuts.

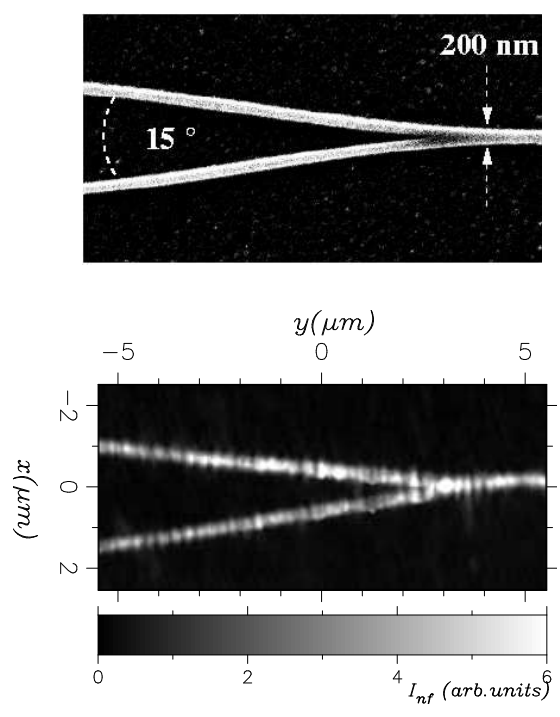

Fig. 5 - Top: Scanning electron microscope image of a $\mathrm{TiO}_{2}$ Y-junction with subwavelength crosssection deposited on glass. Bottom: Near-field optical response of the same Y-junction coupled with a totally reflected focused Gaussian beam (not shown) coming from the right several tens of microns away . 